\title{
Estudo Sobre o Uso de Métricas de Fragmentação de Espectro no Projeto de Algoritmos RSA
}

\author{
Kaio Alexandre da Silva ${ }^{1}$, Ítalo Barbosa Brasileiro ${ }^{2}$, Lucas Rodrigues Costa ${ }^{2}$, \\ André C. Drummond ${ }^{2}$ \\ ${ }^{1}$ Instituto Federal de Educação, Ciência e Tecnologia de Rondônia (IFRO) - \\ Campus Porto Velho Calama \\ CEP 76820-440 - Porto Velho - RO - Brasil \\ ${ }^{2}$ Departamento de Ciência da Computação - Universidade de Brasíla (UnB) \\ Caixa Postal 4466 - 70910-900 - Brasília - DF - Brasil \\ kaio.silva@ifro.edu.br, italo.barbosabrasileiro@yahoo.com.br \\ lucasrc.rodri@gmail.com, andred@unb.br
}

\begin{abstract}
In Elastic Optical Networks (EON), optical paths are established through the arrival of traffic demands. The allocated optical spectrum is adjusted to meet the required bandwidth, which leads to the creation of channels of different capacities in the network. The continuous process of creating and removing these channels causes fragmentation problem, which leads to a non-continuous and non-contiguous distribution of the spectrum, resulting in a reduction in network resource allocation opportunities. This work presents an evaluation of the different spectrum fragmentation metrics, proposed by the EON literature, when they are used as quality measure for resource allocation in Routing and Spectrum Allocation (RSA) algorithms. The results obtained demonstrate that the use of such metrics as the only quality indicator may not be a good strategy in the design of RSA algorithms.
\end{abstract}

Resumo. Nas Redes Ópticas Elásticas (Elastic Optical Networks - EON), os caminhos ópticos são estabelecidos mediante a chegada de demandas de tráfego. O espectro óptico alocado é ajustado para atender à largura de banda necessária, o que leva à criação de canais de diferentes capacidades na rede. O processo contínuo de criação e remoção desses canais causa o problema de fragmentação, o que leva a uma distribuição não contínua e contígua do espectro, resultando na redução das oportunidades de alocação de recursos da rede. Este trabalho apresenta uma avaliação das diferentes métricas de fragmentação de espectro, propostas pela literatura EON, quando são utilizadas como medida de qualidade para alocação de recursos em algoritmos Roteamento e Alocação de Espectro (Routing and Spectrum Allocation - RSA). Os resultados obtidos demonstram que o uso de tais métricas como único indicador de qualidade pode não ser uma boa estratégia no projeto de algoritmos RSA.

\section{Introdução}

As Redes Ópticas Elásticas (EON) surgem como uma solução promissora para o futuro das redes ópticas. Suas características permitem a utilização do espectro óptico de ma- 
neira otimizada [Jinno et al. 2009]. Os intervalos de frequência do espectro óptico são denominados slots, e ao agrupar uma determinada quantidade de slots de maneira contínua e contígua, é estabelecido o canal óptico [Jinno et al. 2009]. Assim é possível estabelecer canais ópticos simultâneos, com diferentes taxas de transmissão de dados. A Figura 1 apresenta em (a) uma EON de 6 nós e 7 enlaces (com 8 slots cada) e em (b) o estado atual dos enlaces.

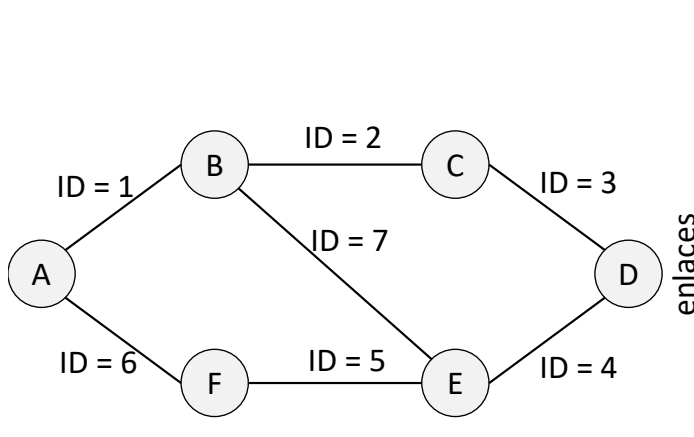

(a)

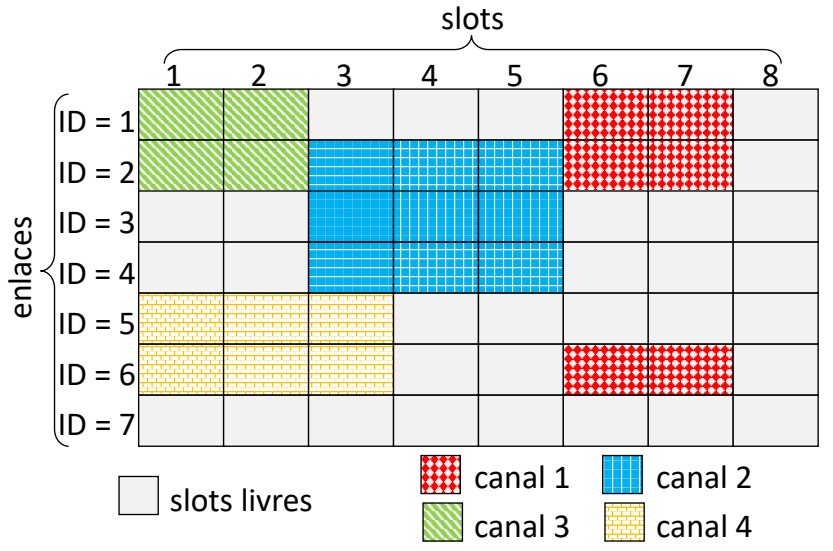

(b)

Figura 1. Um exemplo de EON com 6 nós e 7 enlaces (a), e o estado de utilização do espectro através da representação de slots em cada enlace

A capacidade elástica da EON está associada à possibilidade de alocar canais que ocupam mais de um slot. Assim, ocorre um melhor ajuste no atendimento de diferentes requisitos de largura de banda. A Figura 1 apresenta quatro canais estabelecidos: um canal de dois slots entre os nós A e C (enlaces 1 e 2, canal 3); um canal de três slots entre os nós B e E (enlaces 2, 3 e 4, canal 2); um canal de dois slots entre os nós F e C (enlaces 6, 1 e 2, canal 1); e um canal de três slots entre os nós A e E (enlaces 6 e 5, canal 4). Para o estabelecimento de um canal, é necessário selecionar uma rota com o conjunto adequado de enlaces, juntamente com o intervalo de slots a ser alocado. Esse problema é amplamente discutido na literatura EON e é chamado de Problema de Roteamento e Alocação de Espectro (Routing and Spectrum Allocation - RSA).

É importante destacar que nem sempre é possível escolher uma rota e um intervalo espectral para uma nova solicitação de canal óptico. No exemplo da Figura 1, a chegada de uma nova requisição de três slots entre os nós A e F, independente da rota, resulta em um bloqueio da solicitação, pois não há recursos disponíveis para satisfazer a requisição. A chegada de uma nova requisição de dois slots entre os nós B e C, será bloqueada no enlace 2, mas é possível utilizar os enlaces 7, 4 e 3, e slots 1 e 2 para o estabelecimento do canal óptico. Assim, a organização dos canais já estabelecidos na rede tem forte influência na capacidade de atender novas requisições. A organização dos recursos espectrais é conhecida na literatura como problema de fragmentação, e é explorada e medida de várias formas, como exposto em [Rosa et al. 2012], [Wu et al. 2014] e [Wright et al. 2015].

Para a alocação de um cada canal óptico, o problema RSA deve ser resolvido. Esse problema abrange duas restrições: ( $i$ ) a continuidade; e (ii) a contiguidade do espectro. O primeiro, obriga que os slots escolhidos tenham o mesmo índice em todos os enlaces da rota escolhida. Já o segundo, restringe a alocação de slots de forma ad- 
jacente para compor um canal óptico [Jinno et al. 2009]. Com a criação e a remoção de demandas de tráfego na rede, ao longo do tempo, o espectro torna-se fragmentado, resultando em uma maior probabilidade de bloqueio e uma baixa utilização do uso do espectro [Singh and Jukan 2016]. Este trabalho propõe uma classificação das métricas de fragmentação propostas pela literatura, através de suas características e propriedades. Além disso é proposto uma avaliação da eficiência dessas métricas quando utilizadas como medida de qualidade para alocação de recursos de espectro em uma EON.

\section{Métricas de Fragmentação}

A fragmentação espectral surge como consequência da alocação e remoção de canais ópticos com diferentes larguras de banda. Assim, mesmo que haja quantidade suficiente de slots para atender uma requisição, ela pode ser rejeitada, por não atender as restrições de contiguidade e continuidade do problema RSA [Wright et al. 2015]. As métricas de fragmentação EON são propostas para medir o grau de fragmentação da rede. Identificar uma métrica que forneça uma relação próxima entre a taxa de bloqueio e de fragmentação de espectro garante maior aproveitamento dos recursos espectrais. Entretanto, estabelecer uma relação entre a fragmentação de espectro e a probabilidade de bloqueio de demandas é um problema complexo e têm sido o tema de várias pesquisas relacionadas a EON. Diversos pesquisadores propõem métricas para quantificar a fragmentação de espectro. $\mathrm{O}$ estudo realizado sobre essas métricas levaram à proposição de duas classes de métricas de fragmentação de espectro e suas subdivisões:

1. Quantificam a fragmentação de espectro na rede: Expressam a fragmentação de espectro considerando todos os enlaces presente na EON. Podem ser subdivididas em:

(a) Ciente do Maior Bloco: leva em consideração o maior bloco contíguo de slots livres no enlace, relacionando o maior bloco contíguo livre com a quantidade total de slots livres para mensurar a fragmentação de espectro.

(b) Ciente dos Recursos disponíveis: observa a quantidade de slots disponíveis em cada enlace e calcula quantos canais ópticos poderiam ser alocados.

(c) Ciente da Entropia: leva em consideração a disposição dos slots, realizando, ao mesmo tempo, a análise de contiguidade de espectro e de organização do espectro.

2. Quantificam a fragmentação de espectro no caminho: Expressam a fragmentação de espectro presente no conjunto de enlaces, que venham a formar uma rota entre a origem e o destino da demanda. Podem ser subdivididas em:

(a) Ciente da Demanda: leva em consideração a quantidade de slots que o canal óptico irá necessitar, fazendo a relação entre a demanda e a capacidade disponível.

(b) Consideram Apenas os Recursos Disponíveis: observa a quantidade de slots disponíveis nos enlaces que compõe o futuro caminho óptico que será alocado, independente da demanda solicitada.

Entre as métricas que quantificam a fragmentação de espectro na rede (1), as ciente do maior bloco (1.a) em geral são propostas ruins quando vários enlaces são avaliados, pois a análise independente dos enlaces pode provocar uma avaliação equivocada da real 
fragmentação da rede. Por exemplo, a presença de muitos enlaces pouco fragmentados e poucos enlaces bastante fragmentados podem gerar a conclusão equivocada de que a rede encontra-se muito fragmentada, pois o cálculo é feito através da média de fragmentação dos enlaces. Dentre as métricas ciente dos recursos disponíveis (1.b), a previsão dos canais ópticos é obtida apenas na dimensão de contiguidade de espectro no enlace, não atendendo à característica de continuidade de espectro. Isto torna a predição de alocação de espectro falha e irreal, pois, em muitos casos, a análise de contiguidade de espectro pode determinar que não existe fragmentação de espectro. Entretanto, nenhum canal óptico poderá ser alocado caso necessite de um caminho com mais de um enlace. Por outro lado, em relação as métricas cientes da entropia (1.c), a característica principal dessa estratégia é a capacidade de analisar a organização do espectro, que seria a primeira etapa da análise de continuidade de espectro. Por este fator, espera-se que as métricas dessa subcategoria tenham resultados melhores que as demais.

Entre as métricas que quantificam a fragmentação de espectro no caminho (2), as cientes da demanda (2.a) possuem a característica de que caso uma demanda não possa ser alocada, elas indicam 100\% de fragmentação no caminho, independente da quantidade de slots disponíveis. Em contrapartida, as métricas que consideram apenas os recursos disponíveis (2.b), possuem a característica de que caso seu índice de fragmentação seja $100 \%$, significa que não há nenhuma possibilidade de alocação para qualquer demanda.

A próximas seções apresentam métricas da literatura para o cálculo da fragmentação de espectro na rede. As seguintes definições são importantes para a compreensão das métricas apresentadas:

- Block: conjunto de slots contíguos em uso ou livres;

- LargerFreeBlock: quantidade de slots do maior bloco contíguo livre;

- TotalFreeSlots: total de slots livres;

- NumberSlots: número de slots por enlace;

\subsection{Métricas Ciente do Maior Bloco}

Em [Rosa et al. 2012] e [Thießen and Çavdar 2014] é feita a adaptação da métrica de fragmentação externa tradicional dos discos rígidos [Wilson et al. 1995], denotado pela Equação 1 e chamada de Fragmentação Externa. Calcula-se a relação entre o maior bloco livre e a quantidade total de slots livres.

$$
1-\frac{\text { LargerFreeBlock }}{\text { TotalFreeSlots }}
$$

Para a Equação 1, quanto maior for o valor resultante, maior será a fragmentação presente no enlace. No entanto, ao calcular a média desta métrica, a discrepância dos resultados entre os enlaces não é levada em consideração.

Em [Wu et al. 2014] é proposta uma métrica para calcular o grau de continuidade do espectro através da métrica mostrada na Equação 2.

$$
\frac{\text { LargerFreeBlock }}{\text { TotalFreeSlots }} \times \frac{1}{b_{l}}
$$


A Equação 2 denota o grau de fragmentação da rede, onde $b_{l}$ representa a quantidade de bordas. O autor define que uma borda é a lacuna entre um conjunto de slots, representando um ou mais caminhos ópticos interrompidos. Em outras palavras, as bordas representam caminhos ópticos não adjacentes na fibra óptica. A quantidade de bordas é calculada por meio da Equação 3.

$$
b_{l}=\frac{\left(\sum_{k=1}^{s} S_{l}^{k} \oplus S_{l}^{k+1}+1\right)}{2}
$$

onde, $S_{l}^{k}$ representa o estado do slot $k$ no enlace $l$, sendo 1 quando o slot está ocupado e 0 quando o slot está livre; e $b_{l}$ representa a quantidade de blocos não contíguos presentes no enlace. Assim, quanto maior o valor de $b_{l}$, maior o grau de fragmentação presente no espectro. Para esta métrica, quanto menor o resultado, mais fragmentada é a rede.

\subsection{Métricas Ciente dos Recursos Disponíveis}

Em [Wang et al. 2015] é definida uma métrica para a fragmentação do espectro, que verifica todas as possibilidades de alocação em cada sub-bloco de slots contíguos dentro de cada enlace e executa a multiplicação dessas possibilidades. Esta métrica é ciente da maior granularidade que pode ser atendida na rede e é dividida em duas equações. A Equação 4 é usada para quantificar as possibilidades de alocação em um bloco de slots livres:

$$
A_{n}= \begin{cases}0, & \text { se } n=0 \\ 1+\sum_{i=1}^{n-i} A_{n-i}, & \text { se } 1 \leq n \leq m \\ \sum_{i=1} m A_{n-i}, & \text { se } n>m\end{cases}
$$

na qual $n$ indica a quantidade de slots contíguos, $m$ representa a maior granularidade que pode ser atendida (em número de slots) e $A_{n_{B}}$ representa o número de requisições com tamanho $n$ que podem ser atendidas no bloco de tamanho $B$. A Equação 5 quantifica o grau de fragmentação do espectro multiplicando cada valor de $A_{n_{B}}$ :

$$
\prod_{B \in F} A_{n_{B}}
$$

em que $F$ representa o conjunto de todos os blocos livres na rede, $B$ indica o tamanho de cada bloco e $A_{n_{B}}$ é a chamada para Equação 4. Através da Equação 5 o grau de disponibilidade é obtido, e quanto menor for o resultado, maior será a fragmentação.

Em [Singh and Jukan 2016] é proposta uma métrica de fragmentação de espectro com base no número de requisições que podem ser atendidas utilizando todos os blocos livres no momento atual, divididos pelo número de requisições que seriam atendidas se o espectro fosse totalmente contíguo e livre. A Equação 6, representa a fórmula proposta. 


$$
1-\frac{\sum_{k=1}^{\text {FreeBlocks }}\left(\begin{array}{c}
S_{k}+2 \\
3
\end{array}\right)}{\left(\begin{array}{c}
\text { TotalFreeSlots }+2 \\
3
\end{array}\right)}
$$

$S_{k}$ representa a quantidade de slots do $k$-ésimo bloco livre. Na Equação 6 é verificada a relação da permutação dos blocos livres com o total de slots livres. Para essa métrica, um maior valor está relacionado a uma maior fragmentação.

\subsection{Métricas Ciente da Entropia}

Em [Wright et al. 2015] é proposta uma métrica de fragmentação espectral usando a entropia de Shannon, na qual cada enlace é avaliado com a Equação 7:

$$
-\sum_{i=1}^{N} \frac{D_{i}}{D} \ln \frac{D_{i}}{D}
$$

$N$ indica a quantidade de sub-blocos livres, $D_{i}$ representa o tamanho do sub-bloco atual e $D$ representa a quantidade de slots no espectro. Quando $D_{i}=D$, então $H_{\text {frag }}=0$. Para obter o grau de fragmentação da rede, é feita a soma da relação para todos os enlaces. É importante destacar que essa avaliação prioriza os blocos livres, não importando a distribuição deles nos enlaces. Portanto, quanto maior o resultado, maior a fragmentação.

Em [Altarawneh and Taebi 2015] e [Sugihara et al. 2017] é utilizada a métrica chamada Entropy Usage [Wang and Mukherjee 2012] para calcular a fragmentação do espectro, apresentada na Equação 8. A fragmentação da rede é calculada através da média da fragmentação dos enlaces, e o calculo é composto de quatro etapas:

1. Para cada slot é atribuído 1, se estiver ocupado, e 0 se estiver livre;

2. Para cada par de slots contíguos, se ambos estiverem ocupados ou livres (os dois com o mesmo estado), o valor 0 é atribuído; se apenas um deles estiver ocupado, é atribuído 1 ;

3. Somatório do resultado obtido no passo (2);

4. Aplique a Equação 8.

$$
\frac{\text { step } 3}{\text { NumberSlots }-1}
$$

Para essa métrica, quanto maior o resultado, maior o grau de fragmentação presente na rede. Cada enlace é avaliado separadamente, e ao final é retirada a média para representar a fragmentação de espectro presente na rede.

\section{Metodologia}

O foco deste trabalho é avaliar a eficiência das métricas de fragmentação de espectro em EON, quando utilizadas como medida de qualidade para alocação de recursos em algoritmos RSA. Para fazer isso, todas as métricas são acopladas ao algoritmo RSA $k$-Shortest Path (KSP) [Wan et al. 2011] e exploradas por meio de três estratégias: 
Estratégia 1: Calcula a fragmentação de espectro da rede após alocação da requisição, escolhe a rota com menor, ou maior, impacto na fragmentação de espectro da rede (Figura 2);

Estratégia 2: Calcula a fragmentação de espectro nas $k$ rotas antes da alocação da requisição, busca alocar na rota com a menor fragmentação de espectro (Figura 3);

Estratégia 3: Calcula a fragmentação de espectro nas $k$ rotas após alocação da requisição, realiza a alocação na rota com a menor fragmentação de espectro (Figura 4);

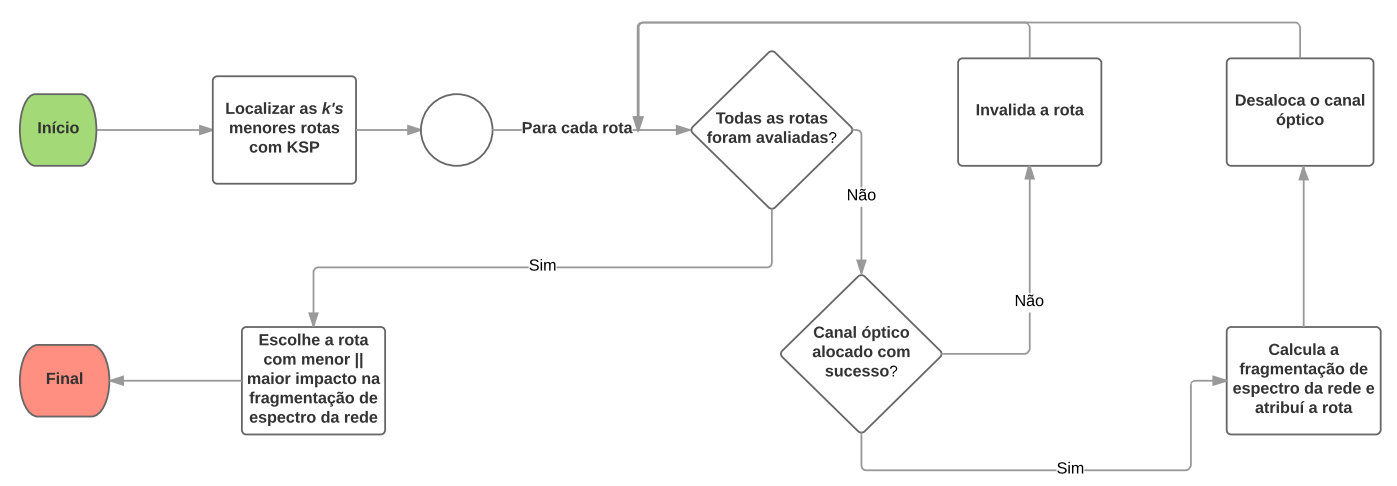

Figura 2. Estratégia da predição de fragmentação de espectro na rede

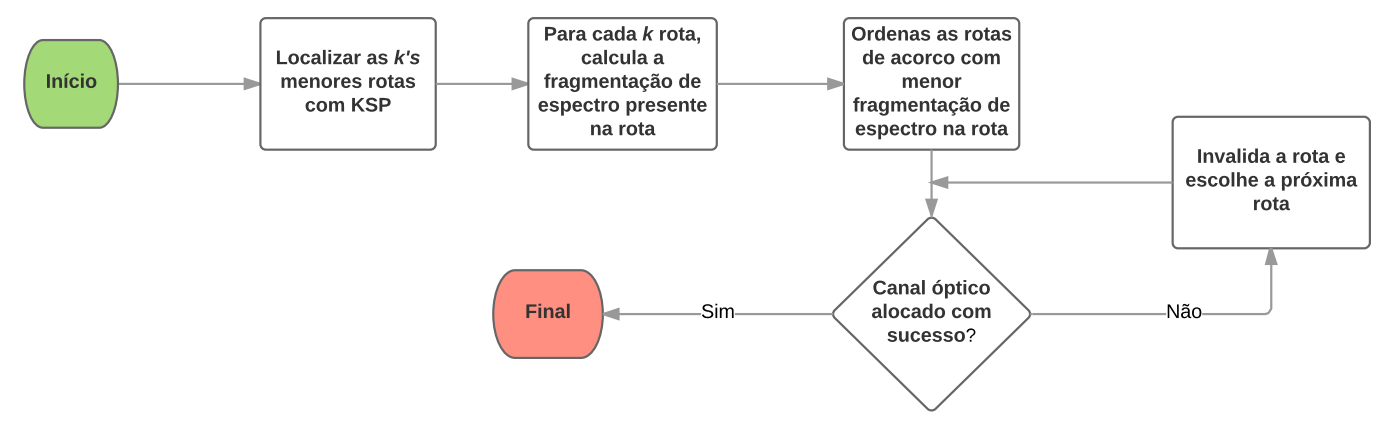

Figura 3. Estratégia de ciente da fragmentação de espectro na rota

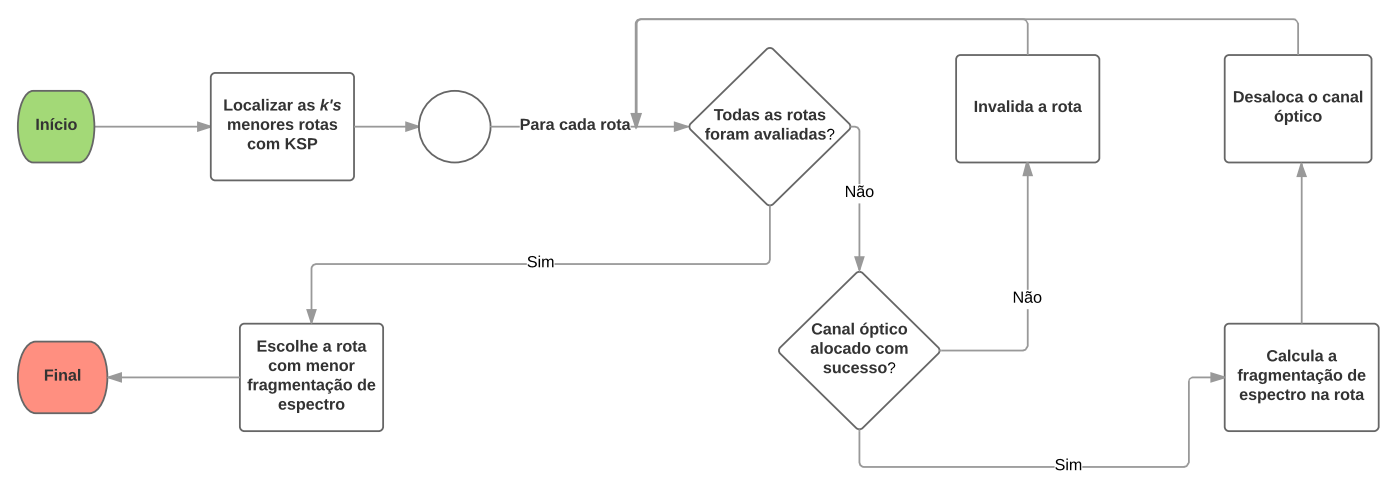

Figura 4. Estratégia da predição de fragmentação de espectro na rota 
A política de alocação utilizada pelo algoritmo KSP foi a First Fit. Sua solução RSA tem duas etapas:

Etapa 1: Roteamento, no qual o algoritmo determina as rotas com as menores $k$ distâncias;

Etapa 2: Alocação do espectro, em que o algoritmo tenta alocar recursos espectrais ao longo da rota escolhida, e se não encontrar recursos livres, ele vai para a próxima rota mais curta, até encontrar recursos disponíveis.

Se não for possível alocar a requisição em nenhuma das $k$ rotas, a requisição será bloqueada. Para este estudo foi considerado $k=8$. A métrica escolhida para medir a eficiência dos algoritmos é a Taxa de Bloqueio de Largura de Banda (BBR). Cada estrategia será testada com todas as métricas apresentadas na Seção 2.

O simulador utilizado foi o Optical Network Simulator - ONS [Costa et al. 2016]. Cada simulação foi realizada cinco vezes utilizando o método de replicações independentes. Para os resultados apresentados foram calculados intervalos de confiança com $95 \%$ de confiabilidade. Cada simulação tem $10^{5}$ requisições de conexões, as quais eram geradas uniformemente com sete tipos de granularidades de largura de banda: $12.5 \mathrm{Gbps}$, 25 Gbps, 50 Gbps, 100 Gbps, 200 Gbps, 400 Gbps e 1 Tbps. O processo de chegada de chamada segue uma distribuição de Poisson com origem e destino uniformemente distribuídos para todos os pares de nós na rede.

A topologia considerada para este estudo foi a USANet, demonstrada na Figura 5. Cada slot possui $12.5 \mathrm{GHz}$ de intervalo de frequência e cada enlace possuindo a capacidade de 320 slots, sendo atribuído um slot como banda de guarda para cada circuito estabelecido. Assume-se que cada transmissor é capaz de transmitir até 80 slots, referente a quantidade de slots necessários para alocar a maior demanda. A modulação considerada foi a $B P S K$, com 1 bit por símbolo.

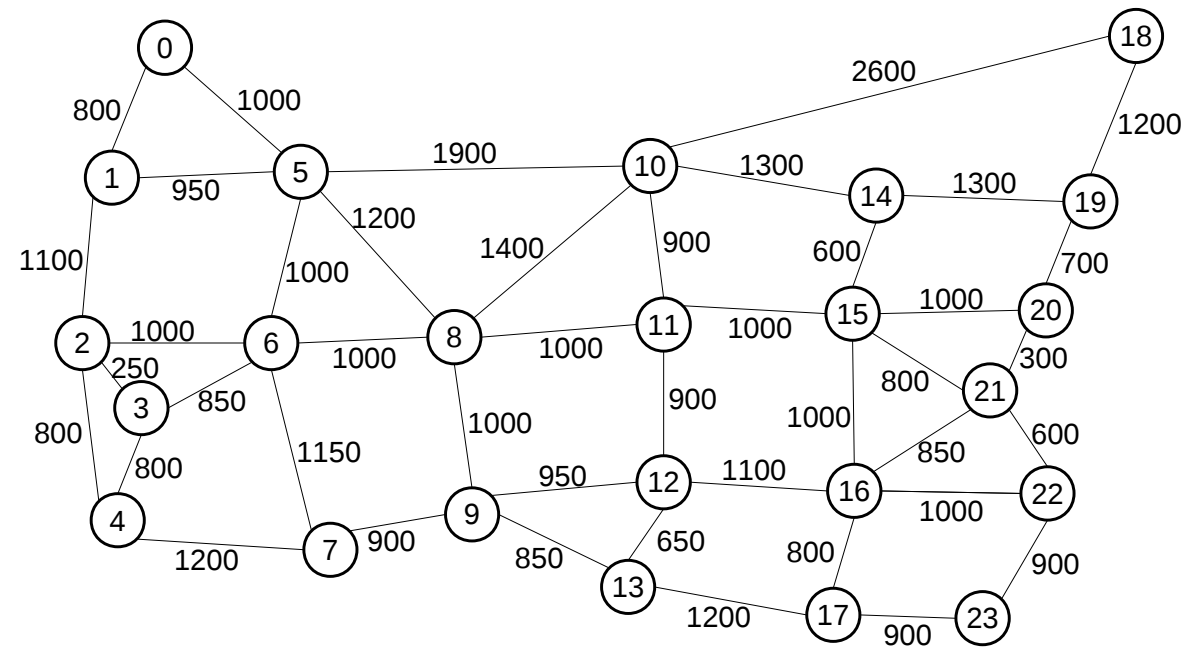

Figura 5. Topologia USANet

\section{Resultados e Discussão}

Os algoritmos baseados nas métricas de fragmentação são identificados com o número da equação da métrica utilizada (Seção 2), enquanto o algoritmo de referência KSP proposto 
por [Wan et al. 2011] é identificado como “KSP Ref”. É necessário deixar claro que o algoritmo KSP Ref não considera qualquer métrica de fragmentação na escolha de caminhos. O BBR reflete o número de requisições bloqueadas, portanto os valores mais baixos são melhores.

\subsection{Estratégia que considera a fragmentação de espectro da rede após a alocação das requisições}

O estudo da predição da fragmentação de espectro na rede, é caracterizado pela identificação da rota que irá diminuir, ou aumentar, a fragmentação de espectro na rede. A Figura 6 mostra a avaliação de desempenho ao escolher a rota que impactará menos a fragmentação da rede, enquanto que a Figura 7 mostra avaliação de desempenho ao escolher a rota que impactará mais a fragmentação da rede.

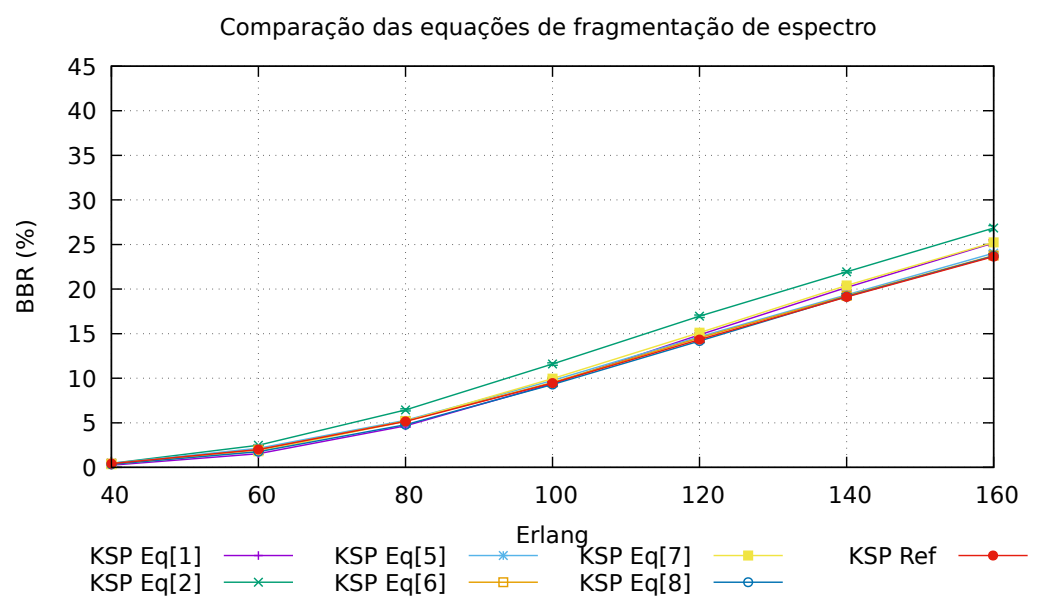

Figura 6. Resultados para escolha da rota com menor impacto na fragmentação de espectro na rede

Os resultados expostos na Figura 6 mostram que a escolha da rota que tem o menor impacto na fragmentação do espectro da rede resulta em um desempenho próximo ao do algoritmo KSP Ref. Apenas a métrica representada pela Equação 2, que considera a quantidade de blocos não-contíguos presentes em cada enlace, obteve um desempenho inferior aos demais, demonstrando que essa é a menos efetiva dentre todas as métricas avaliadas.

Complementando a análise da Figura 6, a Tabela 1 demonstra a média do BBR e a média da fragmentação de espectro da rede de acordo com as métricas estudadas. Para cada uma das estratégias foi verificado a variação da fragmentação de espectro presente na rede em relação ao algoritmo KSP Ref. Com exceção da Equação 8 que obteve desempenho próximo do KSP Ref com a redução da fragmentação. Nesta métrica leva-se em consideração a utilização do espectro não-contíguo presente em cada enlace, fazendo uma análise indireta da continuidade de espectro.

As métricas que levam em consideração o maior bloco livre (Equações 1 e 2) demonstram que não são eficazes ao serem utilizadas para calcular a fragmentação de espectro existente na rede. Isso ocorre porque o cálculo de fragmentação é feito em cada enlace e em seguida é calculado a média dessa fragmentação. A falta de avaliação da 
Tabela 1. Relação entre a média de BBR e média de Fragmentação de Espectro

\begin{tabular}{|c|c|c|c|c|c|c|c|c|c|c|c|c|c|}
\hline \multirow{2}{*}{ Estratégia } & \multirow{2}{*}{ BBR } & \multicolumn{2}{|c|}{ Eq [1] } & \multicolumn{2}{|c|}{ Eq [2] } & \multicolumn{2}{|c|}{ Eq [5] } & \multicolumn{2}{|c|}{ Eq [6] } & \multicolumn{2}{|c|}{ Eq [7] } & \multicolumn{2}{|c|}{ Eq [8] } \\
\hline & & Frag & $\%$ & Frag & $\%$ & Frag & $\%$ & Frag & $\%$ & Frag & $\%$ & Frag & $\%$ \\
\hline KSP Ref & 0,10584855 & 24,3853 & 42857 & 57,05268 & 57143 & 99,20571 & 42857 & 79,26398 & 57143 & 19,23595 & 71429 & 1,44246 & 58571 \\
\hline KSP Eq[1] & 0,10855964 & 21,8902 & $-10,23$ & & & & & & & & & & \\
\hline KSP Eq[2] & 0,12387025 & & & 54,8537 & $+3,85$ & & & & & & & & \\
\hline KSP Eq[5] & 0,10810456 & & & & & 99,9762 & $-0,77$ & & & & & & \\
\hline KSP Eq[6] & 0,10662559 & & & & & & & 79,0817 & $-0,22$ & & & & \\
\hline KSP Eq[7] & 0,11149406 & & & & & & & & & 17,7612 & $-7,66$ & & \\
\hline KSP Eq[8] & 0,10461568 & & & & & & & & & & & 1,3775 & $-4,49$ \\
\hline
\end{tabular}

variação entre os níveis de fragmentação dos enlaces prejudica a avaliação, pois esta não reflete a continuidade do espectro livre da rede.

$\mathrm{Na}$ Figura 7 os resultados apresentam bloqueio mais alto em relação ao resultado do KSP Ref. Isso ocorre porque ao escolher a rota que irá fragmentar mais o espectro na rede, há uma redução da disponibilidade de slots contínuos e contíguos, aumentando assim a probabilidade de bloqueio [Singh and Jukan 2016]. Tanto as métricas que levam em consideração o maior bloco livre (Equações 1 e 2) quanto as métricas que levam em consideração a organização espectral, isto é, a entropia (Equações 7 e 8) demonstram que ao aumentar a fragmentação de espectro, aumenta-se também o BBR. A Tabela 2 expõe os valores da média de BBR e as médias das equações de fragmentação de espectro, observando que em todos os casos com o aumento da fragmentação espectral houve o aumento do BBR.

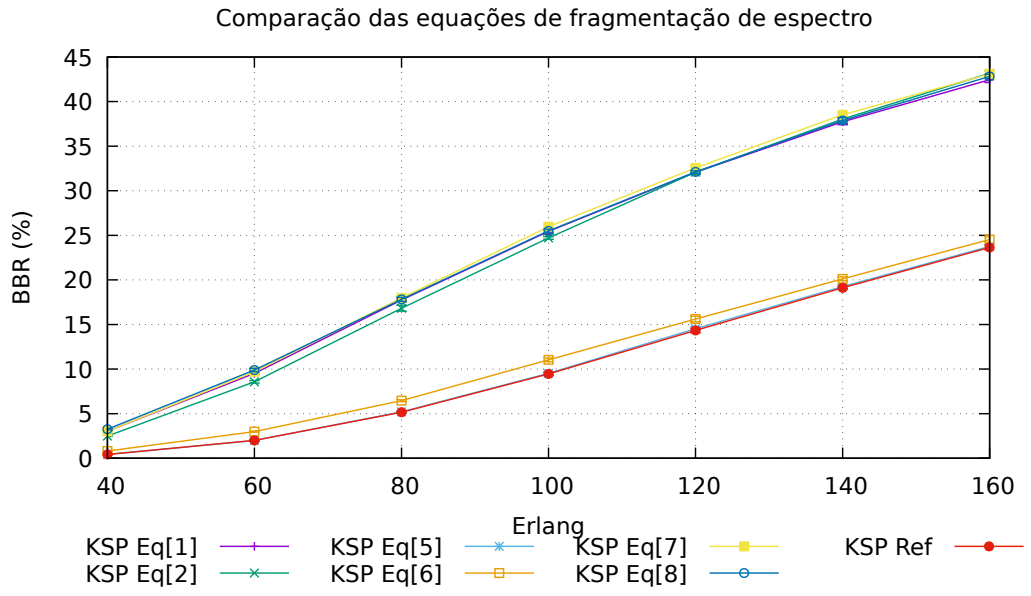

Figura 7. Resultados para escolha da rota com maior impacto na fragmentação de espectro na rede

Com exceção das métricas que levam em consideração os recursos solicitados (Equações 5 e 6), a maioria das métricas levam ao aumento de BBR, em relação ao KSP Ref, quando as rotas com maior impacto de fragmentação espectral são escolhidas. Essas duas equações consideram os recursos disponíveis na rede, e expressam a fragmentação de espectro como a permuta de canais ópticos que podem ser alocados nos enlaces.

Ao analisar as Tabelas 1 e 2 é percebido que a escolha de rotas que levam a menor fragmentação de espectro na rede não é uma estratégia eficaz. A falta de capacidade dessas equações de expressar a fragmentação de espectro na rede dificulta o estabelecimento 
Tabela 2. Relação entre a média de BBR e média de Fragmentação de Espectro

\begin{tabular}{|c|c|c|c|c|c|c|c|c|c|c|c|c|c|}
\hline \multirow{2}{*}{ Estratégia } & \multirow{2}{*}{ BBR } & \multicolumn{2}{|c|}{ Eq [1] } & \multicolumn{2}{|c|}{ Eq [2] } & \multicolumn{2}{|c|}{ Eq [5] } & \multicolumn{2}{|c|}{ Eq [6] } & \multicolumn{2}{|c|}{ Eq [7] } & \multicolumn{2}{|c|}{ Eq [8] } \\
\hline & & Frag & $\%$ & Frag & $\%$ & Frag & $\%$ & Frag & $\%$ & Frag & $\%$ & Frag & $\%$ \\
\hline KSP Ref & 0,10584855 & \multicolumn{2}{|c|}{24,3853142857} & \multicolumn{2}{|c|}{57,0526857143} & \multicolumn{2}{|c|}{99,2057142857} & \multicolumn{2}{|c|}{79,2639857143} & \multicolumn{2}{|c|}{19,2359571429} & \multicolumn{2}{|c|}{1,4424658571} \\
\hline KSP Eq[1] & 0,24001835 & 43,1962 & $+77,14$ & & & & & & & & & & \\
\hline KSP Eq[2] & 0,23696568 & & & 54,8537 & $+42,76$ & & & & & & & & \\
\hline KSP Eq[5] & 0,10662559 & & & & & 99,0400 & $+0,16$ & & & & & & \\
\hline KSP Eq[6] & 0,11649821 & & & & & & & 95,8729 & $+20,95$ & & & & \\
\hline KSP Eq[7] & 0,24407685 & & & & & & & & & 33,0081 & $+71,59$ & & \\
\hline KSP Eq[8] & 0,24182207 & & & & & & & & & & & 2,8084 & $+94,69$ \\
\hline
\end{tabular}

de uma relação confiável com o BBR. Na próxima seção, as métricas são usadas para calcular a fragmentação de espectro presente apenas nas rotas e não na rede inteira.

\subsection{Estratégia que considera a fragmentação de espectro na rota antes da alocação da requisição}

O estudo que traz a ciência da fragmentação de espectro presente na rota é caracterizado pela identificação da fragmentação de espectro presente nas $k$-rotas candidatas. Para isso, é feita a escolha da rota que possui menor (ou maior) fragmentação de espectro. Contudo não há como saber se ao escolher a rota menos (ou mais) fragmentada irá aumentar (ou diminuir) a fragmentação de espectro na rede.

A Figura 8 mostra a avaliação de desempenho ao escolher a rota com menor fragmentação de espectro. As Equações 6 e 7 obtiveram desempenho mais próximo do KSP Ref, pois seus resultados refletem qual rota tem maior continuidade e contiguidade de blocos livres.

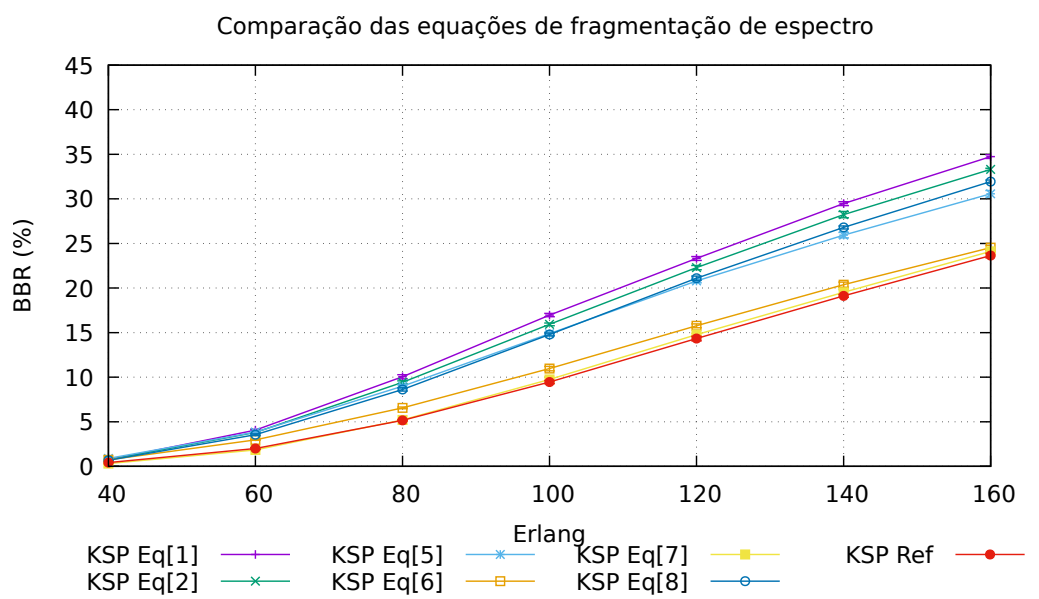

Figura 8. Resultados para escolha da rota com menor fragmentação de espectro

A Tabela 3 mostra que a escolha de rotas com menor fragmentação de espectro não é adequado. A escolha da rota com menor fragmentação espectral, no momento da alocação, faz com que a fragmentação da rede aumente, permitindo a escolha de rotas maiores e resultando em um aumento no BBR.

Os resultados deixam evidentes que a falta da análise da continuidade do espectro leva a uma escolha ruim, pois as $k^{\prime} s$ rotas são ordenadas pela fragmentação espectral. Isso faz com que rotas maiores, ou rotas que não poderiam ser utilizadas por falta de recursos, fossem escolhidas, impactando de forma significativa a fragmentação de espectro da rede. 
Tabela 3. Relação entre a média de BBR e média de Fragmentação de Espectro

\begin{tabular}{|c|c|c|c|c|c|c|c|c|c|c|c|c|c|}
\hline \multirow{2}{*}{ Estratégia } & \multirow{2}{*}{ BBR } & \multicolumn{2}{|c|}{ Eq [1] } & \multicolumn{2}{|c|}{ Eq [2] } & \multicolumn{2}{|c|}{ Eq [5] } & \multicolumn{2}{|c|}{ Eq [6] } & \multicolumn{2}{|c|}{ Eq [7] } & \multicolumn{2}{|c|}{ Eq [8] } \\
\hline & & Frag & $\%$ & Frag & $\%$ & Frag & $\%$ & Frag & $\%$ & Frag & $\%$ & Frag & $\%$ \\
\hline KSP Ref & 0,10584855 & \multicolumn{2}{|c|}{24,3853142857} & \multicolumn{2}{|c|}{57,0526857143} & \multicolumn{2}{|c|}{99,2057142857} & \multicolumn{2}{|c|}{79,2639857143} & \multicolumn{2}{|c|}{19,2359571429} & \multicolumn{2}{|c|}{1,4424658571} \\
\hline KSP Eq[1] & 0,17045862 & 36,4181 & $+49,34$ & & & & & & & & & & \\
\hline KSP Eq[2] & 0,16244493 & & & 76,4257 & $+33,95$ & & & & & & & & \\
\hline KSP Eq[5] & 0,15126093 & & & & & 99,8713 & $-0,67$ & & & & & & \\
\hline KSP Eq[6] & 0,11693428 & & & & & & & 95,0456 & $+19,91$ & & & & \\
\hline KSP Eq[7] & 0,10785416 & & & & & & & & & 22,9492 & $+19,30$ & & \\
\hline KSP Eq[8] & 0,15352351 & & & & & & & & & & & 2,1017 & $+45,70$ \\
\hline
\end{tabular}

\subsection{Estratégia que considera a fragmentação de espectro na rota após a alocação da requisição}

O estudo de predição da fragmentação de espectro na rota, caracteriza-se pela identificação da rota com menor (ou maior) fragmentação espectral após a alocação da requisição. A Figura 9 mostra a avaliação de desempenho considerando a escolha da rota com menor fragmentação de espectro após a alocação da requisição. Nota-se que a Equação 6 se aproxima do resultado do KSP Ref. Isso ocorre porque, ao escolher a rota com maior quantidade de possibilidades de alocação, há uma tendência a escolher as mesmas rotas do KSP Ref.

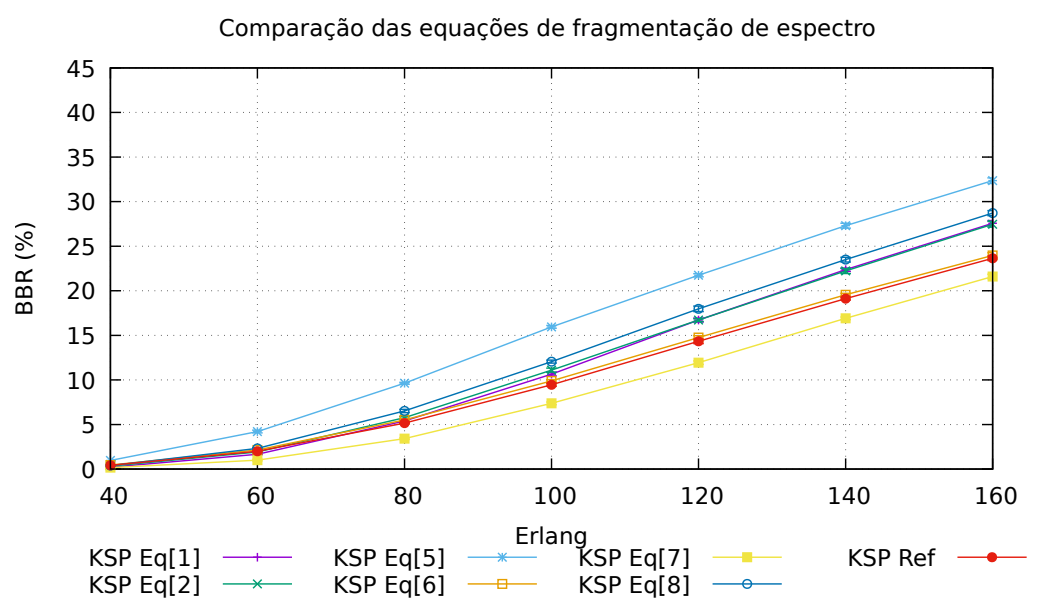

Figura 9. Resultados para escolha da rota com menor fragmentação de espectro após a alocação

Neste cenário a Equação 7 mostra-se como a melhor solução, apresentando resultados melhores que o KSP Ref, exibindo uma eficiência de até $17 \%$ menor em BBR e $0,6 \%$ a menos na fragmentação de espectro na rede. A Tabela 4 demonstra a média da fragmentação de espectro da rede. Nesse cenário, assim como nos anteriores, salienta-se a falta de representatividade das métricas que levam em consideração o maior bloco. Essas estratégias realizam escolhas ruins, pois a escolha de uma rota com menor fragmentação espectral pode levar a escolha de uma rota maior, prejudicando ainda mais a fragmentação espectral na rede.

Nos estudos apresentados, observa-se que a falta de uma equação para retratar a fragmentação de espectro, faz com que as métricas sejam propostas em cenários específicos. Quando são avaliados as métricas em um mesmo cenário, pode-se realmente 
Tabela 4. Relação entre a média de BBR e média de Fragmentação de Espectro

\begin{tabular}{|c|c|c|c|c|c|c|c|c|c|c|c|c|c|}
\hline \multirow{2}{*}{ Estratégia } & \multirow{2}{*}{ BBR } & \multicolumn{2}{|c|}{ Eq [1] } & \multicolumn{2}{c|}{ Eq [2] } & \multicolumn{2}{c|}{ Eq [5] } & \multicolumn{2}{c|}{ Eq [6] } & \multicolumn{2}{c|}{ Eq [7] } & \multicolumn{2}{c|}{ Eq [8] } \\
\cline { 3 - 12 } & & Frag & \% & Frag & \% & Frag & \% & Frag & \% & Frag & \% & Frag & \% \\
\hline KSP Ref & 0,10584855 & 24,3853142857 & 57,0526857143 & 99,2057142857 & 79,2639857143 & 19,2359571429 & 1,4424658571 \\
\hline KSP Eq[1] & 0,12083102 & 26,3724 & $+8,14$ & & & & & & & & & & \\
\hline KSP Eq[2] & 0,12216024 & & & 70,2418 & $+23,11$ & & & & & & & & \\
\hline KSP Eq[5] & 0,16019288 & & & & & 99,7559 & $-0,55$ & & & & & & \\
\hline KSP Eq[6] & 0,10893880 & & & & & & & 87,0866 & $+9,86$ & & & & \\
\hline KSP Eq[7] & 0,08914106 & & & & & & & & & 19,1230 & $-0,58$ & & \\
\hline KSP Eq[8] & 0,13065535 & & & & & & & & & & & 1,7828 & $+23,59$ \\
\hline
\end{tabular}

comprovar, ou validar, a eficiência da proposta. Desta forma, explorar as propostas da literatura através das comparações, possibilita compreender as vantagens e desvantagens, de modo a possibilitar o desenvolvimento de uma equação (ou método) mais representativo.

\section{Considerações finais}

Este artigo investigou o problema da fragmentação de espectro nas redes ópticas elásticas. Foi avaliada a eficiência de várias métricas propostas na literatura, utilizadas como medida de qualidade para alocação de recursos em algoritmos RSA. O problema da fragmentação de espectro é resultado da utilização ineficiente dos recursos da rede, e causa degradação na performance da rede, isto é, aumenta a probabilidade de bloqueio de largura de banda. Uma maior fragmentação de espectro está relacionada a uma maior probabilidade de bloqueio. Logo, estabelecer uma relação entre a fragmentação de espectro e a probabilidade de bloqueio de largura de banda é o tema de várias pesquisas relacionadas à EON.

Através das análises foi constatado que as equações propostas na literatura, não são representativas o suficiente a ponto de expressar o grau de fragmentação da rede, pois elas realizam a avaliação individual dos enlaces, contemplando apenas a característica da contiguidade do espectro presente nos enlaces analisados. Contudo, foi observado que uma boa representação da fragmentação do espectro EON não deve somente contemplar as características de contiguidade do espectro como também a continuidade do espectro na rede. Portanto, a medir a fragmentação por meio de cálculos como média, ou produto, é ineficaz.

Como trabalhos futuros pretende-se aumentar as estratégias de estudo, avaliando também as métricas que quantificam a fragmentação de espectro no caminho, realizando análises combinadas com outras políticas de alocação. Além disso, serão realizadas simulações em outras topologias, de maneira a possibilitar o aperfeiçoamento das políticas de alocação, das métricas de fragmentação de espectro e dos algoritmos RSA.

\section{Agradecimentos}

Os autores agradecem ao Instituto Federal de Educação, Ciência e Tecnologia de Rondônia - campus Porto Velho Calama por disponibilizar a infra-estrutura do Centro Internacional de Métodos Numéricos em Engenharia (Sala IFRO-CIMNE) no desenvolvimento deste trabalho.

\section{Referências}

Altarawneh, L. and Taebi, S. (2015). Bandwidth granularity adaptation for multipath provisioning in elastic optical ofdm-based networks. In 2015 IEEE International Conference on Electro/Information Technology (EIT), pages 236-240. 
Costa, L. R., de Sousa, L. S., de Oliveira, F. R., K. A. Silva, P. J. S. J., and Drummond, A. C. (2016). Ons: Simulador de eventos discretos para redes opticas wdm / eon. XXXIV Simpósio Brasileiro de Redes de Computadores e Sistemas Distribuídos, pages 1195-1202.

Jinno, M., Takara, H., Kozicki, B., Tsukishima, Y., Sone, Y., and Matsuoka, S. (2009). Spectrum-efficient and scalable elastic optical path network: architecture, benefits, and enabling technologies. IEEE Communications Magazine, 47(11):66-73.

Rosa, A., Cavdar, C., Carvalho, S., Costa, J., and Wosinska, L. (2012). Spectrum allocation policy modeling for elastic optical networks. In High Capacity Optical Networks and Emerging/Enabling Technologies, pages 242-246.

Singh, S. K. and Jukan, A. (2016). Non-disruptive spectrum defragmentation with holding-time awareness in optical networks. In 2016 International Conference on Optical Network Design and Modeling (ONDM), pages 1-6.

Sugihara, S., Hirota, Y., Fujii, S., Tode, H., and Watanabe, T. (2017). Dynamic resource allocation for immediate and advance reservation in space-division-multiplexing-based elastic optical networks. IEEE/OSA Journal of Optical Communications and Networking, 9(3):183-197.

Thießen, C. and Çavdar, (2014). Fragmentation-aware survivable routing and spectrum assignment in elastic optical networks. In 2014 6th International Workshop on Reliable Networks Design and Modeling (RNDM), pages 97-101.

Wan, X., Wang, L., Hua, N., Zhang, H., and Zheng, X. (2011). Dynamic routing and spectrum assignment in flexible optical path networks. In 2011 Optical Fiber Communication Conference and Exposition and the National Fiber Optic Engineers Conference, pages $1-3$.

Wang, N., Jue, J. P., Wang, X., Zhang, Q., Cankaya, H. C., and Sekiya, M. (2015). Holding-time-aware scheduling for immediate and advance reservation in elastic optical networks. In 2015 IEEE International Conference on Communications (ICC), pages 5180-5185.

Wang, R. and Mukherjee, B. (2012). Spectrum management in heterogeneous bandwidth networks. In 2012 IEEE Global Communications Conference (GLOBECOM), pages 2907-2911.

Wilson, P. R., Johnstone, M. S., Neely, M., and Boles, D. (1995). Dynamic storage allocation: A survey and critical review. In Proceedings of the International Workshop on Memory Management.

Wright, P., Parker, M. C., and Lord, A. (2015). Minimum- and maximum-entropy routing and spectrum assignment for flexgrid elastic optical networking [invited]. IEEE/OSA Journal of Optical Communications and Networking, 7(1):A66-A72.

Wu, J., Zhang, M., Wang, F., Yue, Y., and Huang, S. (2014). An optimal independent sets based greedy spectral defragmentation algorithm in elastic optical network. In 2014 13th International Conference on Optical Communications and Networks (ICOCN), pages $1-4$. 